\title{
Burkitt-type lymphoma incidentally found as the cause of acute appendicitis: a case report and review of literature
}

\author{
Davit Shahmanyan 1,7, Brian Saway 2,7, Hannah Palmerton 3,7 , John S. Rudderow ${ }^{4}$, Christopher M. Reed ${ }^{5,7}$, \\ Terri-Ann Wattsman ${ }^{4}$, Emily R. Faulks ${ }^{4}$, Bryan R. Collier ${ }^{4}$, Robert E. Budin ${ }^{4}$ and Mark E. Hamill ${ }^{4,6,7^{*}}$ (D)
}

\begin{abstract}
Background: Appendectomy remains one of the most common emergency operations. Recent research supports the treatment of uncomplicated appendicitis with antibiotics alone. While nonoperative management of appendicitis may be safe in some patients, it may result in missed neoplasms. We present a case of acute appendicitis where the final pathology resulted in a diagnosis of a Burkitt-type lymphoma.

Case presentation: An 18-year-old male presented to the emergency department with $24 \mathrm{~h}$ of right lower quadrant pain with associated urinary retention, anorexia, and malaise. Past medical history was significant for intermittent diarrhea and anal fissure. He exhibited focal right lower quadrant tenderness. Workup revealed leukocytosis and CT uncovered acute appendicitis with periappendiceal abscess and no appendicolith. Laparoscopic appendectomy was performed and found acute appendicitis with associated abscess abutting the rectum and bladder. Pathology of the resected appendix reported acute appendicitis with evidence of Burkitt-type lymphoma. A PET scan did not reveal any residual disease. Hematology/oncology was consulted and chemotherapy was initiated with an excellent response.

Conclusions: Appendiceal lymphomas constitute less than $0.1 \%$ of gastrointestinal lymphomas. Primary appendix neoplasms are found in $0.5-1.0 \%$ of appendectomy specimens following acute appendicitis. In this case, appendectomy allowed for prompt identification and treatment of an aggressive, rapidly fatal lymphoma resulting in complete remission.
\end{abstract}

Keywords: Burkitt lymphoma, Acute appendicitis, Surgical pathology, Incidental finding

\section{Background}

While acute appendicitis is a common pathological entity, it is a surgical emergency. The lifetime risk of developing appendicitis ranges from 7 to $8 \%$, with the median age of diagnosis between 10 and 11 years of age [1]. The pathogenesis of acute appendicitis centers around luminal obstruction leading to inflammation, rising intraluminal pressure, and ultimately ischemia. Consequently,

\footnotetext{
${ }^{*}$ Correspondence: hamillm@mac.com

${ }^{6}$ Department of Surgery, University of Nebraska Medical Center, 983280

NE Medical Center, MSB 4553, Omaha, NE 68198-3280, USA

Full list of author information is available at the end of the article
}

the appendix enlarges and leads to inflammatory changes in nearby tissue. While luminal obstruction is the ubiquitous primary event that sets off this inflammatory sequence of events, the cause of luminal obstruction includes fecalith, lymphoid hyperplasia, foreign bodies, and cancer [2]. While appendiceal lymphomas constitute $0.015 \%$ of gastrointestinal lymphoma cases, primary appendix neoplasms are found in $0.5-1.0 \%$ of appendectomy specimens following acute appendicitis [3].

Effective management of appendicitis is a topic of debate, with considerable options for operative and nonoperative approaches. Nonoperative management has 
become increasingly popular in recent years. A recent study demonstrated $4.9 \%$ of cases in 2014 were managed nonoperatively, which represents a $4.7 \%$ increase per year since 1998 [4]. While nonoperative management of acute uncomplicated appendicitis has a success rate of $75 \%$ at 1 year, we argue that operative management should not be hastily disregarded in this disease [5].

\section{Case presentation}

Our patient was an 18-year-old male who presented to the emergency department with a complaint of approximately $24 \mathrm{~h}$ of pelvic pain. The symptoms were worsening and associated with urinary retention, nausea, and anorexia. The pain was exacerbated by movement. He reported similar symptoms approximately 1 month prior, with a negative workup by his primary care physician at that time. Past medical history was significant for intermittent loose stools, anal fissures, and a prior cyst in his neck. Surgical history included a colonoscopy as part of a workup for hematochezia several years earlier, which diagnosed an anal fissure. Excision of a reportedly benign neck cyst was also completed previously; however, no pathology was available.

On presentation, he was a well-appearing young adult male in mild distress. He was afebrile, and his vital signs were within normal limits, without evidence of tachycardia, tachypnea, or hypotension. His abdomen was soft but tender to palpation in the right lower quadrant, without evidence of generalized peritonitis. Lab values were insignificant except for leukocytosis (WBC 17.4 k) and mild hyperglycemia (blood glucose level $124 \mathrm{mg} /$ dl). Computed tomography imaging obtained prior to the surgical consult demonstrated dilation of the appendix up to $11 \mathrm{~mm}$ with periappendiceal fluid (Fig. 1) and a 3-cm abscess adjacent to the appendiceal tip (Fig. 2) consistent with appendicitis with perforation and locally contained abscess.

Treatment options included appendectomy, treatment with antibiotics, and percutaneous drainage of the abscess, all of which were discussed with the patient and his family. After considering options, the patient and team elected to proceed to the operating room for laparoscopic appendectomy and drainage of the abscess. Antibiotic treatment with piperacillin/tazobactam (Zosyn) was initiated and the patient brought to the operating room and placed under general anesthesia for the procedure. Operative findings included purulent fluid throughout the peritoneal cavity with the appendix laying down in the pelvis. Appendiceal inflammation with obvious perforation to the appendiceal tip and adjacent abscess was noted. The patient's postoperative course was significant for persistent nausea for $12 \mathrm{~h}$ after the procedure, which was treated with antiemetics and resolved.

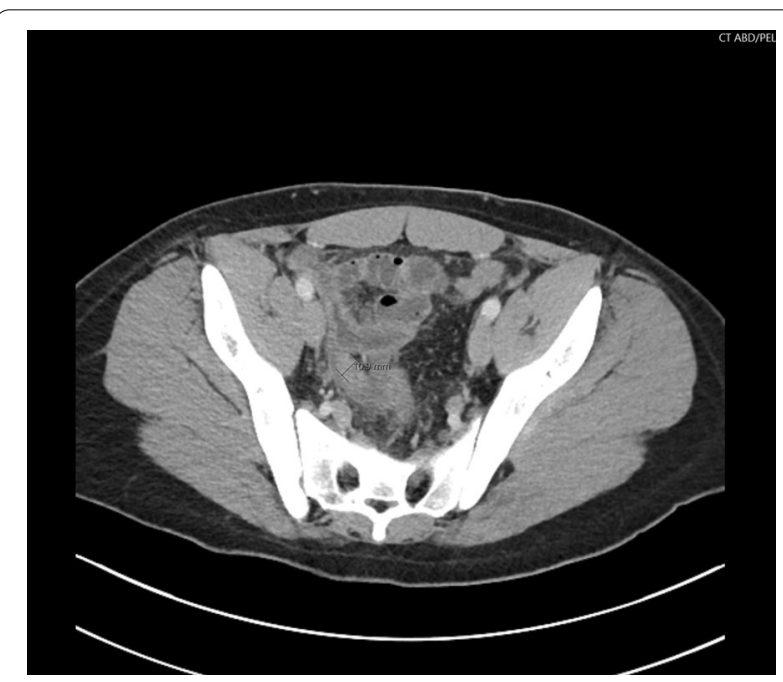

Fig. 1 Transverse view of the abdominal CT demonstrating 0.9-mm dilated appendix with periappendiceal fluid in the pelvis

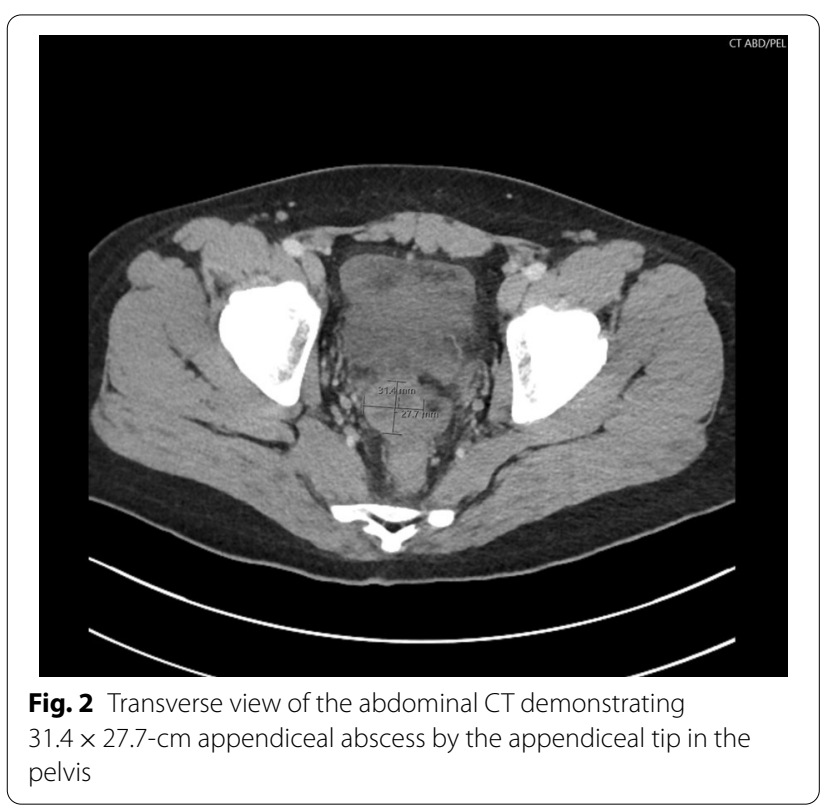

He was discharged to home on postoperative day \#1 with a plan to complete a 10-day course of oral amoxicillin/ clavulanic acid (Augmentin).

Several hours after his discharge, an urgent call was received from the pathologist reviewing the appendix specimen. Surprisingly, the appendix demonstrated a high grade transmural lymphoid malignancy with extra-appendiceal deposits (Figs. 3 and 4). The pathological diagnosis was initially classified as Burkitt lymphoma, which was later reclassified based on additional pathologic staining as a B-cell lymphoma with features 


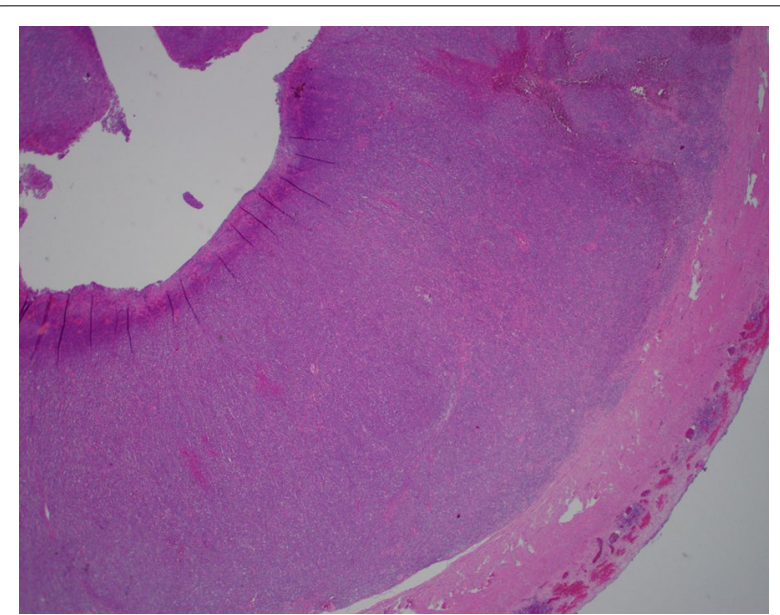

Fig. 3 Hematoxylin and eosin staining of appendiceal tissue demonstrating significant lymphoid invasion ( $\times 20$ magnification)

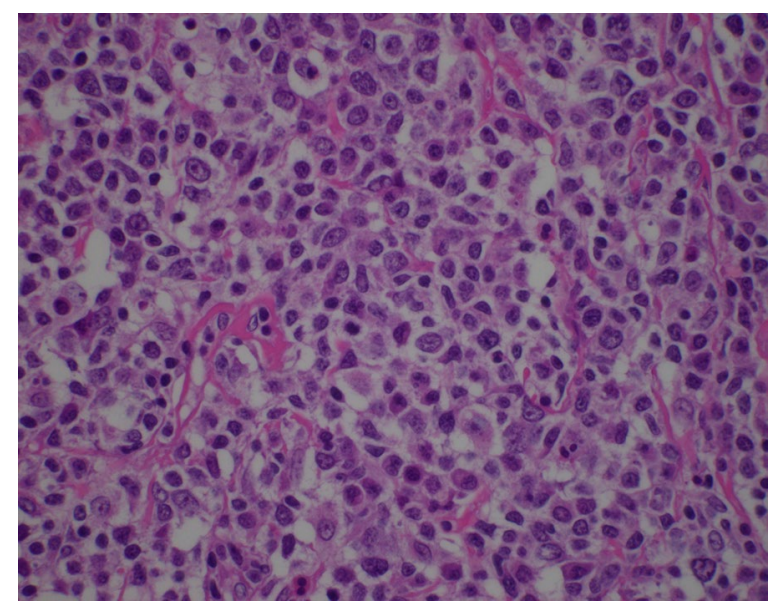

Fig. 4 Hematoxylin and eosin staining of appendiceal tumor (x600 magnification)

between diffuse large B-cell lymphoma and Burkitt lymphoma. Positive tumor markers included CD20 (Fig. 5), CD10 (Fig. 6), BCL6, and Ki67. Urgent surgical follow-up and hematology/oncology consultation was obtained.

Given the Burkitt-type features and the potentially rapid progression of a Burkitt lymphoma, the decision was made to treat as a confirmed Burkitt lymphoma. The patient promptly underwent PET scanning, lumbar puncture, and port placement. PET imaging revealed no evidence of distant disease. He also had sperm banking performed to preserve the potential for future fertility. After sperm banking, the patient underwent a total of four cycles of cyclophosphamide, vincristine, doxorubicin, high-dose methotrexate, ifosfamide, etoposide, high-dose cytarabine (CODOX-M/IVAC) chemotherapy

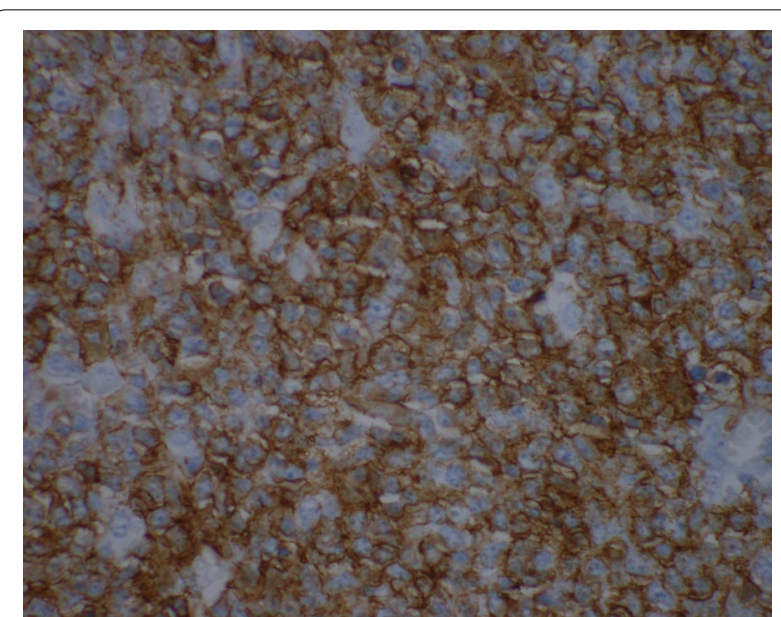

Fig. 5. $\times 600$ Magnification of appendiceal tissue with CD20 immunohistochemistry staining

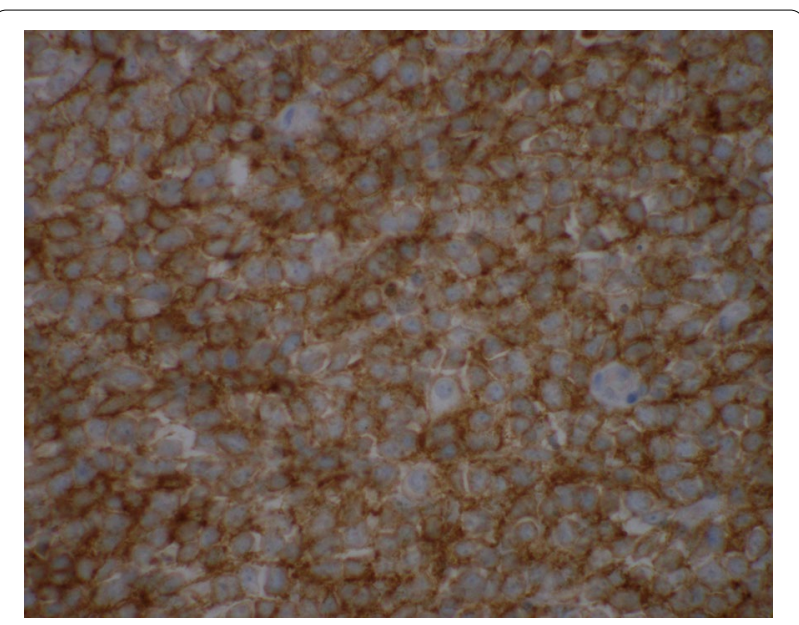

Fig. 6. $\times 600$ Magnification of appendiceal tissue with CD10 immunohistochemistry staining

with an excellent response. Now more than 30 months out from treatment, he remains in complete remission.

\section{Discussion}

The gastrointestinal tract is the most common extranodal sites of lymphoma metastases, accounting for $5-20 \%$ of all cases [6]. Primary GI lymphoma, however, is exceedingly rare and constitutes only about $1-4 \%$ of all GI malignancies as the majority occurs secondary to widespread nodal disease [7]. While this disease can manifest in any part of the GI tract, the most frequent sites of occurrence are the stomach, small intestine, and colorectal region. Of the three locations, the colorectal region, including the appendix, is the least common site constituting $6-12 \%$ of GI lymphomas [8]. While the 
clinical presentation and epidemiology of primary lymphoma of the colorectal region has been well studied, primary appendiceal lymphoma is a rare entity that is not yet clinically and prognostically well defined. The largest study to date assessed 116 patients with primary appendiceal lymphoma with a mean age at diagnosis of 48 years [3]. The study also found that males and Caucasians were significantly more affected, and diffuse large B-cell lymphoma was the most common histologic subtype (34.5\%). Burkitt lymphoma is the second most common cause of appendiceal lymphoma. It is a subtype of non-Hodgkin lymphoma that is highly aggressive and rapidly fatal if left untreated. Patients with Burkitt lymphoma generally do not present with classic B-symptoms suggestive of malignancy, making it commonly found later in its clinical course, with subsequently poor outcomes [9]. Additionally, in Burkitt lymphoma of sporadic origin, presenting abdominal symptoms may mimic that of acute appendicitis. When the lymphoma presents within the appendix, the resulting acute appendicitis and appendectomy with histological analysis provide clinicians with an early diagnosis and an opportunity for early treatment $[4,10,11]$.

Interestingly, patients with Burkitt lymphoma of the appendix presented at an earlier age (33 years) compared with follicular lymphoma (59 years) and diffuse large B-cell lymphoma (53 years), which is consistent with our case involving a young white male patient. The mortality rate of primary appendiceal lymphoma is promising, as the 5 -year survival rate was found to be $67 \%$ in the cohort studied. Similarly, the 5-year survivor rate of Burkitt lymphoma/leukemia is $87 \%$ in ages $0-19$ and $60 \%$ in those 20-39 [12]. The clinical presentation widely varies as the patients can present with symptoms of intestinal obstruction and GI bleeding, or it can manifest as acute appendicitis as the expansion of tissue leads to obstruction and subsequent inflammation and rupture of the appendix. For primary appendiceal lymphoma, early diagnosis and definitive treatment are essential as delay in treatment can lead to local and lymphatic spread of the disease, leading to increased mortality. In this case, there was some question of the final pathologic diagnosis as the lymphoma had staining characteristics which were somewhat atypical. This is not uncommon and has led to a reclassification of these tumor types [13]. However, due to the aggressive and fatal nature of untreated Burkitt lymphoma the decision was made by the oncologists to proceed with rapid and aggressive treatment assuming typical Burkitt lymphoma features were present.

CT Imaging is a vital tool for diagnosis of appendiceal neoplasms by demonstrating the disproportionately increased diameter of the appendix shown on CT-scans $(>3 \mathrm{~cm})$. In contrast, non-tumoral appendicitis does not have as large a diameter. CT Imaging is also critical in assessing the extent of tumor burden throughout the body [14]. However, in the case of primary appendiceal lymphoma presenting as acute appendicitis, the decision to utilize CT is still controversial. While CT-scanning grants over $90 \%$ sensitivity and specificity for acute appendicitis and helps with other differential diagnoses, it is not mandatory as a diagnosis of acute appendicitis can be made clinically [8].

\section{Discussion regarding medical decision-making we made during the case}

Timely appendectomy, either open or laparoscopic, has been the recommended treatment of choice for acute appendicitis due to recent advances in perioperative management, which has lowered rates of wound infection, shortened hospital stays, and significantly decreased morbidity [15]. However, recent research has provided evidence for nonoperative management of acute appendicitis. To date, six randomized trials have compared antibiotics with appendectomy for nonperforated appendicitis in adults, reporting a reduction in leukocytosis, avoidance of peritonitis, and general symptom reduction with antibiotic treatment alone. Furthermore, these studies state that $90 \%$ of patients treated with antibiotics can avoid surgery during the initial admission, and $70 \%$ of those successfully treated with antibiotics during the initial admission can avoid surgery during the 1st year [5, 16-20]. Unfortunately, only one study analyzed follow-up data beyond the 1st year of treatment of acute appendicitis with antibiotics and found a $39.1 \%$ incidence of recurrent appendicitis at 5 years [21]. The Comparison of Outcomes of antibiotic Drugs and Appendectomy (CODA) randomized controlled study, which is ongoing, aims to examine if antibiotic therapy is non-inferior to surgery, and will have follow-up of 2 years to see if any harm results from nonoperative therapy [22]. While this research provides a compelling argument for consideration of antibiotics alone for treatment of acute appendicitis, there is more recent literature which speaks to the concern of whether treatment with antibiotics alone can lead to missed or delay in diagnoses of primary neoplasms. This would have been the case in our report. For adults the risk of an appendiceal cancer is not insignificant in those presenting with appendicitis. Lu et al. found when reviewing National Surgical Quality Improvement Program (NSQIP) data, that in a cohort of 21,069 patients from 2016 to 2017 who underwent appendectomy for either imaging-proven appendicitis or equivocal for appendicitis imaging, that the odds ratio of being diagnosed with cancer increased with each decade after age 50 up to age 80 [23]. Skendelas et al. found a $0.53 \%$ incidence of appendiceal malignancies in NSQIP database patients who had undergone appendectomy for 
appendicitis from 2010 to 2018, and 1.7\% incidence in their local patients who had surgery at two hospitals in The Bronx, New York City [24]. Finally, the recent PeriAppendicitis Acuta randomized trial looking at the necessity of interval appendectomy after successful nonoperative treatment of peri-appendicular abscess was terminated early basic on ethical concerns after a neoplasm rate of $20 \%$ was discovered in patients who underwent interval appendectomy [25].

\section{Conclusion}

While new research has provided evidence for antibiotics alone as treatment for acute appendicitis, we believe it will lead to missed or delayed diagnoses of primary appendix neoplasms. Our patient discussed above presented with acute appendicitis, he was treated with appendectomy and was diagnosed with Burkitt-type lymphoma of the appendix on pathology. Due to the early diagnosis, this patient was able to be rapidly and effectively treated resulting in a complete remission and a significant improvement in his prognosis. Had he been treated with antibiotics alone, even if it was successful in the control of his appendicitis, the chances of a poor outcome from a later diagnosis of Burkitt lymphoma is very high. This potential risk needs to be considered when examining the risks and benefits of operative versus nonoperative management of acute appendicitis.

\section{Abbreviations \\ CT: Computed tomography; PET: Positron emission tomography; CODOX-M/ IVAC: Cyclophosphamide, vincristine, doxorubicin, high-dose methotrexate/ ifosfamide, etoposide, high-dose cytarabine; Gl: Gastrointestinal; WBC: White blood cell.}

\section{Acknowledgements}

The authors would like to acknowledge the Virginia Tech Open Access Subvention fund for providing payment of the article processing charges for the publication of this manuscript.

\section{Authors' contributions \\ DS prepared the manuscript for submission, provided the design of the final report, provided edits to, and discussed contents of the manuscript. BS described and designed the original report. CR, T-AW, JR, RB, BC, EF, and HP provided edits and discussed the contents of the manuscript. MH provided the case report, prepared the manuscript for submission, and edited the manuscript throughout the process. All authors read and approved the final manuscript.}

\section{Funding}

This study was not funded.

\section{Availability of data and materials}

All data generated or analyzed during this study are included in this published article.

\section{Declarations}

Ethics approval and consent to participate

Per the federal definition of research and IRB protocols at the Carilion Clinic, this report consisted of one patient for which consent was exempted. The exception criteria included: the review was done by persons involved in the patient's care, information was deidentified, no changes were made in the patient's care, no testing was added for the purpose of reporting the case, and there was no investigational drug, device, or biologic used.

\section{Consent for publication}

Per the federal definition of research and IRB protocols at the Carilion Clinic, this report consisted of one patient for which consent was exempted. The exception criteria included: the review was done by persons involved in the patient's care, information was deidentified, no changes were made in the patient's care, no testing was added for the purpose of reporting the case, and there was no investigational drug, device, or biologic used.

\section{Competing interests}

The authors declare no potential conflicts of interest.

\section{Author details}

${ }^{1}$ Department of Surgery, University of California San Francisco Fresno, 2823 Fresno St, Fresno, CA 93701, USA. ${ }^{2}$ Department of Neurosurgery, Medical University of South Carolina, 96 Jonathan Lucas Street, Charleston, SC 29425, USA. ${ }^{3}$ Department of Surgery, Madigan Army Medical Center, 9040A Jackson Ave, Joint Base Lewis-McChord, WA 98431, USA. ${ }^{4}$ Department of Surgery, Virginia Tech Carilion School of Medicine and Research Institute, 3 Riverside Circle, Roanoke, VA 24016, USA. ${ }^{5}$ Valley Health Bariatric and Metabolic Surgery Program, 1870 Amherst St, Winchester, VA 22601, USA. ${ }^{6}$ Department of Surgery, University of Nebraska Medical Center, 983280 NE Medical Center, MSB 4553, Omaha, NE 68198-3280, USA. ${ }^{7}$ Present Address: Virginia Tech Carilion School of Medicine and Research Institute, 3 Riverside Circle, Roanoke, VA 24016, USA.

Received: 27 April 2021 Accepted: 25 August 2021

Published online: 24 September 2021

\section{References}

1. de Morais SD, Mikhael BM, Németh SIA, Paulo IML, de Barros ÉOH, Lima OAT. Burkitt's lymphoma presenting as acute appendicitis: a case report. J Surg Case Rep. 2018;2018:1-3.

2. Petroianu A, Vinicius Villar Barroso T. Pathophysiology of acute appendicitis. JSM Gastroenterol Hepatol. 2016. https://www.jscimedcentral.com/ Gastroenterology/gastroenterology-4-1062.pdf.

3. Ayub A, Santana-Rodríguez N, Raad W, Bhora FY. Primary appendiceal lymphoma: clinical characteristics and outcomes of 116 patients. J Surg Res. 2017;207:174-80

4. Horn CB, Tian D, Bochicchio GV, Turnbull IR. Incidence, demographics, and outcomes of nonoperative management of appendicitis in the United States. J Surg Res. 2018;223:251-8.

5. Gonzalez DO, Deans KJ, Minneci PC. Role of non-operative management in pediatric appendicitis. Semin Pediatr Surg. 2016;25:204-7. https://doi. org/10.1053/.sempedsurg.2016.05.002.

6. Freeman C, Berg JW, Cutler SJ. Occurrence and prognosis of extranodal lymphomas. Cancer. 1972;29:252-60.

7. Ghimire P, Wu G-Y, Zhu L. Primary gastrointestinal lymphoma. World J Gastroenterol. 2011;17:697-707.

8. Parks NA, Schroeppel TJ. Update on imaging for acute appendicitis. Surg Clin N Am. 2011;91:141-54.

9. Vons C, Barry C, Maitre S, Pautrat K, Leconte M, Costaglioli B, et al. Amoxicillin plus clavulanic acid versus appendicectomy for treatment of acute uncomplicated appendicitis: an open-label, non-inferiority, randomised controlled trial. Lancet (Lond, Engl). 2011;377:1573-9.

10. Khanna M, Buddhavarapu SR. Primary Burkitt's lymphoma of the appendix presenting as acute abdomen: a case report. J Radiol Case Rep. 2008;2:9-14.

11. Salminen P, Tuominen R, Paajanen $H$, Rautio T, Nordström P, Aarnio M, et al. Five-year follow-up of antibiotic therapy for uncomplicated acute appendicitis in the APPAC randomized clinical trial. JAMA. 2018;320:1259. https://doi.org/10.1001/jama.2018.13201.

12. Costa LJ, Xavier AC, Wahlquist AE, Hill EG. Trends in survival of patients with Burkitt lymphoma/leukemia in the USA: an analysis of 3691 cases. Blood. 2013;121:4861-6. https://ashpublications.org/blood/article/121/ 24/4861/31627/Trends-in-survival-of-patients-with-Burkitt. 
13. Thomas DA, O'Brien S, Faderl S, Manning JT, Romaguera J, Fayad L, et al. Burkitt lymphoma and atypical Burkitt or Burkitt-like lymphoma: should these be treated as different diseases? Curr Hematol Malig Rep. 2011;6:58-66.

14. Abdalla MF, El-Hennawy HM. Unusual presentation for primary appendiceal lymphoma: a case report. Indian J Surg. 2010;72:289-92.

15. Hansson J, Körner U, Khorram-Manesh A, Solberg A, Lundholm K Randomized clinical trial of antibiotic therapy versus appendicectomy as primary treatment of acute appendicitis in unselected patients. Br J Surg. 2009;96:473-81.

16. Körner H, Söndenaa K, Söreide JA, Andersen E, Nysted A, Lende TH, et al. Incidence of acute nonperforated and perforated appendicitis: agespecific and sex-specific analysis. World J Surg. 1997;21:313-7.

17. Styrud J, Eriksson S, Nilsson I, Ahlberg G, Haapaniemi S, Neovius G, et al. Appendectomy versus antibiotic treatment in acute appendicitis. A prospective multicenter randomized controlled trial. World J Surg. 2006;30:1033-7.

18. Shelton T, McKinlay R, Schwartz RW. Acute appendicitis: current diagnosis and treatment. Curr Surg. 2003;60:502-5.

19. Salminen $P$, Paajanen $H$, Rautio T, Nordström $P$, Aarnio M, Rantanen $T$, et al. Antibiotic therapy vs appendectomy for treatment of uncomplicated acute appendicitis. JAMA. 2015;313:2340-8. https://doi.org/10.1001/jama. 2015.6154.

20. Eriksson S, Granström L. Randomized controlled trial of appendicectomy versus antibiotic therapy for acute appendicitis. Br J Surg. 1995;82:166-9.

21. Salminen P, Tuominen R, Paajanen $H$, Rautio T, Nordström P, Aarnio M, et al. Five-year follow-up of antibiotic therapy for uncomplicated acute appendicitis in the APPAC randomized clinical trial. JAMA J Am Med Assoc. 2018;320:1259-65.

22. Davidson GH, Flum DR, Talan DA, Kessler LG, Lavallee DC, Bizzell BJ, et al. Comparison of Outcomes of antibiotic Drugs and Appendectomy (CODA) trial: a protocol for the pragmatic randomised study of appendicitis treatment. BMJ Open. 2017;7:e016117.

23. Lu P, McCarty JC, Fields AC, Lee KC, Lipsitz SR, Goldberg JE, et al. Risk of appendiceal cancer in patients undergoing appendectomy for appendicitis in the era of increasing nonoperative management. J Surg Oncol. 2019;120:452-9.

24. Skendelas JP, Alemany VS, Au V, Rao D, McNelis J, Kim PK. Appendiceal adenocarcinoma found by surgery for acute appendicitis is associated with older age. BMC Surg. 2021;21:228. https://doi.org/10.1186/ s12893-021-01224-0.

25. Mällinen J, Rautio T, Grönroos J, Rantanen T, Nordström P, Savolainen $\mathrm{H}$, et al. Risk of appendiceal neoplasm in periappendicular abscess in patients treated with interval appendectomy vs follow-up with magnetic resonance imaging. JAMA Surg. 2019;154:200. https://doi.org/10.1001/ jamasurg.2018.4373.

\section{Publisher's Note}

Springer Nature remains neutral with regard to jurisdictional claims in published maps and institutional affiliations.

\section{Submit your manuscript to a SpringerOpen ${ }^{\odot}$ journal and benefit from:}

- Convenient online submission

- Rigorous peer review

- Open access: articles freely available online

- High visibility within the field

- Retaining the copyright to your article

Submit your next manuscript at $\boldsymbol{\nabla}$ springeropen.com 